Revista Perspectivas Online: Exatas \& Engenharia Fevereiro/2020, v. 10, n. 27, p. $27-41$ ISSN: 2236-885X (Online) DOI: $10.25242 / 885 X 102720201952$

\title{
ANÁLISIS DE LA FALLA REINCIDENTE EN UN ENGRANAJE HELICOIDAL
}

\section{Bárbara Ferreira de Oliveira ${ }^{1 *}$, Alexandre Alves Pessanha1, Luis Augusto Hernandez Terrones $^{2}$}

\begin{abstract}
RESUMEN
SILVA, F.; SILVA, B. Análisis de la falla reincidente en un engranaje helicoidal. Perspectivas Online: Exatas \& Engenharias, v. 10, n. 27, p. 27 - 41, 2020.

Este artículo presenta el análisis de falla de un engranaje helicoidal perteneciente a un reversor de un motor de combustión interna de tipo marítimo. Después de analizar el principio de funcionamiento, las causas potenciales de fallas $y$ dimensionamiento de este componente fueron seleccionadas y cortadas muestras del material del engranaje para análisis visual y microestructural, así como para ensayos mecánicos de dureza y microdureza. También fueron calculadas las tensiones de flexión y de contacto para confirmar si están dentro de la norma. Los

resultados mostraron que el material utilizado para la fabricación del engranaje es un acero ASTM 8620, constituido microestructuralmente por un núcleo martensítico y por una superficie cementada, cuyos espesor y dureza se encuentran adecuados a los patrones exigidos. Aunque las propiedades metalúrgicas y mecánicas del acero del engranaje estuvieran apropiadas, se verificó que la tensión de flexión actuante es mayor que su valor admisible, estando por esta razón susceptible a una falla por sobrecarga.
\end{abstract}

Palabras clave: Microstructura; Tensión; Acero; Sobrecarga.

Persp. Online: exatas \& eng., Campos dos Goytacazes, 27 (10) 27 - 41 - 2020

seer.perspectivasonline.com.br 


\begin{abstract}
This paper presents the failure analysis of a helical gear belonging to a sea-type internal combustion engine reverser. First, the operating principles, potential failure causes, and component sizes were analyzed; gear material samples were then obtained for visual and microstructural analysis and mechanical testing of hardness and micro-hardness. Bending and contact stresses were then calculated to

that the gear was fabricated of ASTM 8620 steel, which is constituted of a martensitic core and a carburized surface with a thickness and hardness well within the standards. Although the metallurgical and mechanical properties of the gear's steel were appropriate, it was verified that the acting bending stress is greater than its admissible value, being for this reason susceptible to an overload failure.
\end{abstract} verify standard compliance. Results reveal

Keywords: Microstructure, Stress, Hardness, Overload.

${ }^{1}$ Institutos Superiores de Ensino do CENSA - ISECENSA - Laboratório de Análise e Projeto de Sistemas Mecânicos LAPSIM - Rua Salvador Correa, 139, Centro, Campos dos Goytacazes, RJ, CEP: 28035-310, Brasil;

${ }^{2}$ Universidade Estadual do Norte Fluminense Darcy Ribeiro - UENF - Laboratório de Materiais Avançados - LAMAV/CCT Av. Alberto Lamego, 2000, Parque Califórnia, Campos dos Goytacazes, RJ, CEP: 28013-602, Brasil.

(*) e-mail: barbara.fo@gmail.com

Data de recebimento: 12/12/2019. Aceito para publicação: 19/02/2020.

Persp. Online: exatas \& eng., Campos dos Goytacazes, 27 (10) 27 - 41 - 2020

seer.perspectivasonline.com.br 


\section{INTRODUCCIÓN}

Los engranajes helicoidales son ampliamente utilizados en la industria como elementos de transmisión de potencia entre ejes paralelos o cruzados, debido a que el nivel de ruido atribuido a su operación es mínimo, las cargas soportadas pueden ser considerablemente altas y también son capaces de soportar cargas dinámicas (ASI, 2005).

Todos los engranajes son diseñados considerando sus posibles modos de falla. La Asociación Americana de fabricantes de engranajes (AGMA) menciona 21 modos de falla en esos componentes, siendo más común las fallas que ocurren por fractura por sobrecargas o fatiga del material por esfuerzos en condiciones desfavorables, efectos por la deficiencia de lubricación o por condiciones de altas temperaturas y velocidades elevadas (FUJITA et al., 1982; KU, 2008).

Las propiedades mecánicas de los engranajes son tan importantes que pueden definir el modo de falla bajo el cual el engranaje está más propenso. Por ejemplo, un engranaje más duro está más propenso a la fractura de los dientes mientras que el que presenta menor dureza está más susceptible a fatiga por contacto. Es importante mencionar que las propiedades mecánicas y el comportamiento en servicio de estos componentes son fuertemente dependientes de la microestructura, una vez que su deficiencia resultante del uso incorrecto del material o de un tratamiento térmico inadecuado puede conducir a una falla prematura (ASM HANDBOOK COMMITEE, 1990; ASM HANDBOOK COMMITEE, 2002; BROOKS, 2002; ROY, OOI, e SUNDARARAJAN, 2018; ROY e SUNDARARAJAN, 2019).

Generalmente, los materiales más utilizados en engranajes de componentes mecánicos son aceros de baja aleación, tales como: ASTM 4026, ASTM 8620 y ASTM 4620. Aunque se recomienda los tratamientos de normalizado o recocido subcrítico de los aceros para la fabricación del engranaje, posteriormente son sometidos a un tratamiento termoquímico de endurecimiento superficial, como la cementación, seguida de templado y revenido. Aun así, aunque la superficie del engranaje sea endurecida, la capacidad de la carga soportada por el engranaje debe ser calculada llevando en cuenta la fatiga de contacto (ASM HANDBOOK COMMITEE, 2002; LIU et al., 2018; FUJITA et al., 1982).

Entre las tensiones actuantes durante el trabajo de los engranajes, se destaca la tensión de flexión en el pie del diente, pues esta puede causar la fractura del diente. De esta manera, mediante su valor se define si el engranaje está en condiciones de soportar los esfuerzos de transmisión, siendo necesario que sea menor, si es aplicado un factor de seguridad o igual a la tensión de flexión admisible del material indicado (MELCONIAN, 2009).

En este trabajo fueron evaluados las propiedades mecánicas, la microestructura, las tensiones, así como las condiciones de operación de un engranaje helicoidal de un bote salvavidas que sufrió fractura de los dientes durante el uso de la caja de transmisión de la cual es componente, con el objetivo de verificar si atendían las recomendaciones y normas exigidas.

\section{METODOLOGIA}

Para realizar este trabajo fue utilizado un engranaje de un bote salvavidas de un motor de combustión interna de tipo marítimo con tres cilindros. Las especificaciones del motor son descritas en la Tabla 1.

Persp. Online: exatas \& eng., Campos dos Goytacazes, 27 (10) 27 - 41 - 2020

seer.perspectivasonline.com.br 
Tabla 1: Especificaciones del motor

\begin{tabular}{cc}
\hline & Motor \\
\hline Potencia continua & $50 \mathrm{cv}(38 \mathrm{~W})$ a $2500 \mathrm{rpm}$ \\
Torque continuo & $152 \mathrm{Nm}(15,5 \mathrm{kgf.m})$ a $1800 \mathrm{rpm}$ \\
Potencia intermitente & $56 \mathrm{cv}(41 \mathrm{~W})$ a $2500 \mathrm{rpm}$ \\
Torque intermitente & $167,8 \mathrm{Nm}(17,1 \mathrm{kgf.m})$ a $1800 \mathrm{rpm}$ \\
\hline
\end{tabular}

En la Figura 1 se observa la fractura de la mayoría de los dientes durante la prueba de navegación del bote salvavidas.

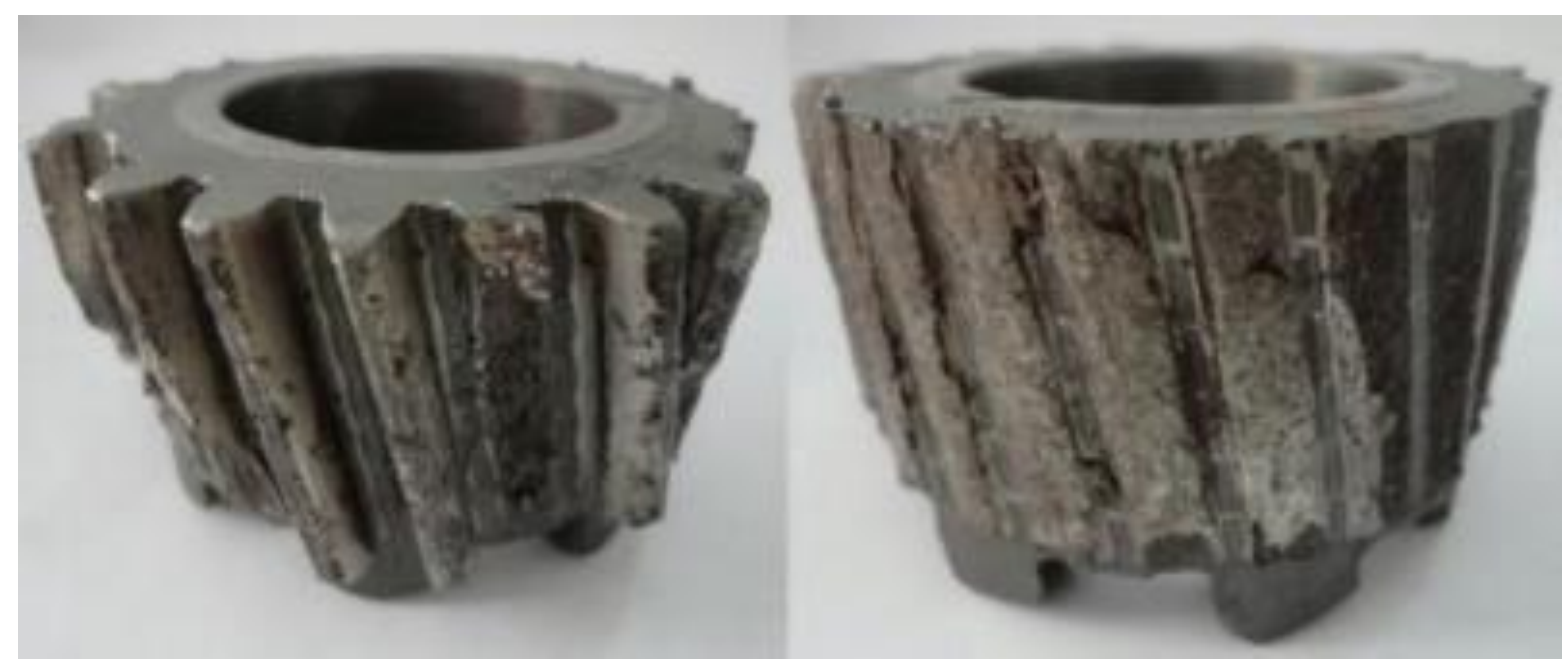

Figura 1: Engranaje estudiado

De acuerdo con el histórico de reposición después de la falla del engranaje, objeto de este estudio, el mismo siempre fue substituido siguiendo una norma de material y tratamiento termoquímico, siendo compuesta por un acero ASTM 8620, cementado y templado obteniendo una superficie endurecida de aproximadamente $1 \mathrm{~mm}$ de profundidad. La norma ANSI/AGMA 2001-D04 clasifica los aceros en grados 1, 2 y 3, siendo creciente el nivel de acuerdo con la calidad del material. Considerando que no fueron realizadas muchas exigencias con relación al acero utilizado y al proceso de fabricación del engranaje, el material estudiado fue tratado como grado 1.

Para determinar las dimensiones del engranaje fue utilizado un calibrador analógico con graduación de 0,05 $\mathrm{mm}-1 / 128$ ", con exactitud de $+/-0,05 \mathrm{~mm}$, además de un goniómetro analógico universal de marca INSIZE, con lupa $360^{\circ}$, libre de paralaje. Las dimensiones obtenidas y los cálculos determinados son presentados en la Tabla 2.

Persp. Online: exatas \& eng., Campos dos Goytacazes, 27 (10) 27 - 41 - 2020

seer.perspectivasonline.com.br 
Tabla 2: Dimensiones del piñon y de la corona

\begin{tabular}{|c|c|c|}
\hline & Piñon & Corona \\
\hline M - Módulo (mm) & \multicolumn{2}{|c|}{3} \\
\hline$D_{e}$ - Diámetro interno $(\mathrm{mm})$ & 64 & 137,5 \\
\hline$D_{i}$ - Diámetro externo $(\mathrm{mm})$ & 76,8 & 151 \\
\hline$d$ - Diámetro primitivo $(\mathrm{mm})$ & 69 & 141 \\
\hline$N$ - Número de dientes & 23 & 47 \\
\hline$N_{n}$ - Número equivalente de dientes & 33,41 & 68,28 \\
\hline$\psi$ - Ángulo de la hélice (grados) & \multicolumn{2}{|c|}{28} \\
\hline$\phi$ - Ángulo de presión (grados) & \multicolumn{2}{|c|}{14} \\
\hline$\phi_{n}$ - Ángulo de presión normal (grados) & \multicolumn{2}{|c|}{12,42} \\
\hline$h_{t}-$ Profundidad total $(\mathrm{mm})$ & \multicolumn{2}{|c|}{6,4} \\
\hline$b_{w}-$ Ancho de la cara $(\mathrm{mm})$ & \multicolumn{2}{|c|}{32} \\
\hline$t_{o}-$ Espesor del diente en el tope $(\mathrm{mm})$ & \multicolumn{2}{|c|}{2,2} \\
\hline$t_{r}-$ Espesor del aro debajo del diente $(\mathrm{mm})$ & \multicolumn{2}{|c|}{11} \\
\hline$P_{d}-$ Paso diametral $(\mathrm{mm})$ & \multicolumn{2}{|c|}{0,33} \\
\hline$P_{n}$ - Paso normal $(\mathrm{mm})$ & \multicolumn{2}{|c|}{10,2} \\
\hline$P_{c}-$ Paso circular $(\mathrm{mm})$ & \multicolumn{2}{|c|}{9,42} \\
\hline$P_{c n}-$ Paso circular normal (mm) & \multicolumn{2}{|c|}{8,32} \\
\hline$P_{a}-$ Paso axial $(\mathrm{mm})$ & \multicolumn{2}{|c|}{17,72} \\
\hline$c_{d}-$ Distancia central $(\mathrm{mm})$ & \multicolumn{2}{|c|}{105} \\
\hline
\end{tabular}

\subsection{Análisis de la composición química}

La composición química del acero fue determinada en un espectrómetro marca Thermo y modelo ARL4460.

\subsection{Análisis Visual}

A pesar de ser una técnica simple, él ensayo de inspección visual es muy importante no solamente para detectar las fallas en la superficie, sino que también evaluar posibles distorsiones en la estructura, acabado superficial y de geometría de un engranaje. Fue aplicado el método de análisis visual a simple vista para caracterizar las fracturas y marcas de degaste como consecuencia de esfuerzos sufridos por el engranaje.

\subsection{Obtención de muestras}

Para realizar los ensayos de dureza, microdureza y análisis metalográfico fueron obtenidas muestras cortando el engranaje utilizando disco abrasivo de partículas de alúmina. Primeramente, el corte del engranaje fue realizado en la dirección transversal y luego perpendicularmente exponiendo el material en la parte central de la pieza. Después del corte las muestras fueron embebidas en baquelita. 


\subsection{Análisis Microestructural}

Para el análisis metalográfico se utilizaron muestras obtenidas en la sección transversal a los dientes del engranaje. El lijado fue realizado con lijas de agua con granulometrías de 180, 220, 320, 400, 600 y 1200 mesh cambiando la dirección de lijado en $90^{\circ}$ en cada cambio de granulometría. El pulimiento de las muestras fue realizado con alúmina en suspensión con $1 \mu \mathrm{m}, 0,5 \mu \mathrm{m}$ y $0,3 \mu \mathrm{m}$. El ataque químico con el objetivo de revelar la superficie endurecida fue realizado mediante una solución picral $5 \%$ durante 15 segundos y para revelar la microestructura fue utilizada una solución de Nital $2 \%$. La obtención de imágenes de la microestructura fue realizada en el microscopio confocal Olympus OLS 4000 Confocal Laser.

\subsection{Ensayo de microdureza Vickers}

Las medidas de microdureza fueron realizadas a lo largo de la superficie en la región del adendo, del dedendum y en el núcleo del diente. El equipo de microdureza utilizado fue Shimadzu HMV. Para este ensayo de microdureza se utilizó una carga de $1 \mathrm{kgf}$ durante 10 segundos. La distancia entre las indentaciones fue de $0,2 \mathrm{~mm}$ y la primera indentación fue realizada a una distancia de $0,3 \mathrm{~mm}$ de la superficie para evitar errores.

\subsection{Ensayo de dureza Rockwell C}

En ensayo de dureza Rockwell C fue realizado en un durómetro Pantec con una precarga de $10 \mathrm{kgf}$ y una carga de $150 \mathrm{kgf}$ de acuerdo con la norma ASTM E18. Fueron realizados cinco medidas en la superficie y en el nucleó de los dientes del engranaje.

\subsection{Cálculo de las tensiones de flexión y de contacto}

Las tensiones de flexión y de contacto fueron calculadas de acuerdo con la norma ANSI/AGM 2001-D04, cuyas ecuaciones utilizadas son 1 y 2, respectivamente.

$$
\sigma_{t}=\frac{W_{t} \cdot P_{d} \cdot K_{o} \cdot K_{s} \cdot K_{m} \cdot K_{\vartheta} \cdot K_{b}}{b_{w} \cdot Y_{h}}
$$

donde:

$W_{t}$ : carga tangencial;

$K_{o}$ : factor de sobrecarga.

$K_{s}$ : factor de tamaño. La ANSI/AGMA 2001 - D04 especifica que este factor es igual a 1 para a mayoría de engranajes, con excepción de engranajes muy grandes o anchos. Para este estudio, fue considerado $K_{s}=1$;

$K_{m}$ : factor de distribución de carga;

$K_{\vartheta}$ : factor dinámico;

$K_{b}$ : Fator de espesor. Este factor es igual a 1 cuando la relación entre el espesor del anillo y el ancho del orificio sea mayor que 1,2, que es el caso del engranaje estudiado; $Y_{h}$ : factor de corrección geométrico (flexión), definido por la norma AGMA 908-B89.

$$
\sigma_{c}=p_{h}\left(K_{o} \cdot K_{s} \cdot K_{m} \cdot K_{\vartheta}\right)^{\frac{1}{2}}
$$

donde: $p_{h}$ : tensión máxima de contacto Hertziana.

Persp. Online: exatas \& eng., Campos dos Goytacazes, 27 (10) 27 - 41 - 2020 seer.perspectivasonline.com.br 


\section{RESULTADOS}

\subsection{Análisis químico}

En la Tabla 3 son presentados los resultados de la composición química del material, así como también los valores establecidos para el acero ASTM 8620 de acuerdo con la norma ASTM A29. El material para fabricar el engranaje obedece a las especificaciones exigidas, siendo apropiado para engranajes de transmisiones marítimas según Davis (2002).

Tabla 3: Composición química del material (\% p.) y establecida por ASTM A29 para acero ASTM 8620

\begin{tabular}{ccc}
\hline Elemento (\%p) & Material estudiado & ASTM 8620 (ASTM A29) \\
\hline Carbono (C) & 0,20 & 0,18 a 0,23 \\
Manganeso (Mn) & 0,80 & 0,70 a 0,90 \\
Fósforo (P) & 0,031 & Máximo de 0,035 \\
Azufre (S) & 0,038 & Máximo de 0,040 \\
Silicio (Si) & 0,27 & 0,15 a 0,35 \\
Níquel (Ni) & 0,55 & 0,40 a 0,70 \\
Cromo (Cr) & 0,54 & 0,40 a 0,60 \\
Molibdeno (Mo) & 0,23 & 0,15 a 0,25 \\
\hline
\end{tabular}

\subsection{Análisis macrográfico del engranaje}

A través de un análisis macroscópico de las fracturas de los dientes del engranaje estudiado, como muestra la Figura 2, fueron observados aspectos que caracterizan una ruptura por sobrecarga, como señales de una ruptura fibrosa y también evidencias de haber sido "rasgada".

Fue observado también un astillamiento de los dientes como consecuencia de impactos causados por un engranamiento deficiente.

No fueron encontradas marcas características de fractura de los dientes por flexión por fatiga, Alban (1985), como modo de falla más común en engranajes. Generalmente, estas fallas surgen como resultado del desarrollo de una grieta en la superficie del pie del diente cuyo lado está cargado y es caracterizado como una superficie lisa y uniforme de la raíz después de la retirada del diente. 


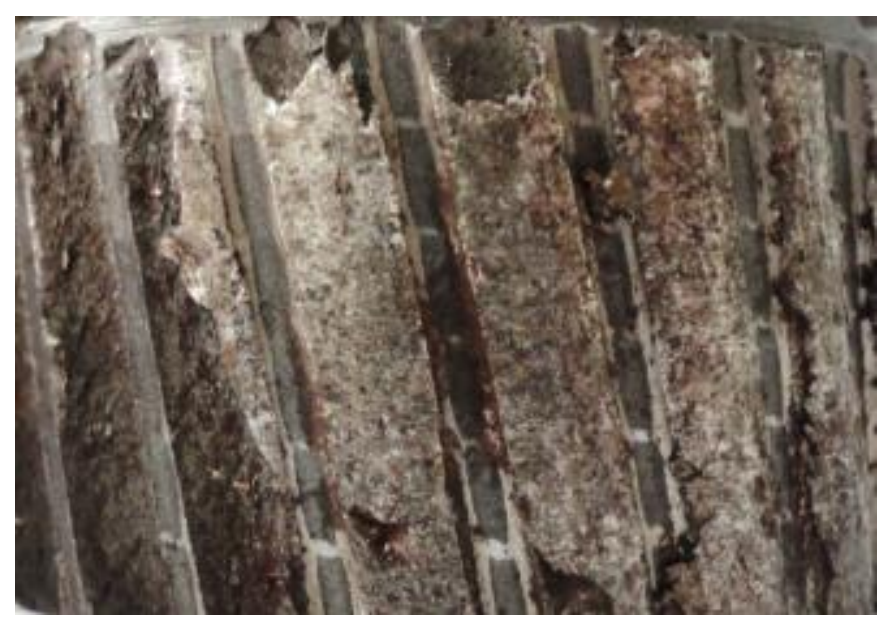

Figura 2: Superficie de fractura del engranaje estudiado

En la Figura 3 se presenta una macrografía de la muestra atacada con picral $5 \%$. Se puede observar que el material presenta un perfil uniforme de endurecimiento. Como la mayoría de los engranajes son sometidas a tensiones cíclicas y las tensiones nominales son más altas en la superficie, es necesario realizar tratamientos para aumentar la resistencia al desgaste y a la fatiga, ya que las grietas por fatiga son casi siempre originadas en la superficie. Como resultado de estos tratamientos se obtiene un núcleo tenaz asociado a una superficie endurecida, En el caso del engranaje estudiado, este fue sometido a un endurecimiento por cementación, que, para ser considerado satisfactorio, deberá resultar en una alta dureza superficial, alta resistencia de contacto, alta resistencia al desgaste, distribución adecuada de las tensiones compresivas, alta resistencia a la flexión, así como una baja tasa de propagación de picaduras (pitting).

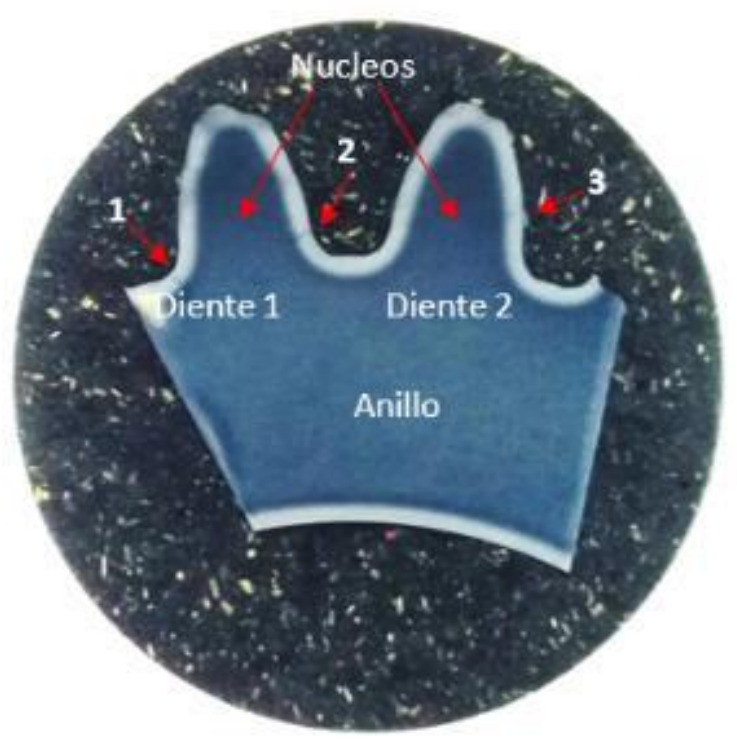

Figura 3: Macrografía de la muestra seleccionada del engranaje.

Persp. Online: exatas \& eng., Campos dos Goytacazes, 27 (10) 27 - 41 - 2020

seer.perspectivasonline.com.br 


\subsection{Caracterización microestructural de los dientes del engranaje}

En las Figuras 4 (a) y (b) son mostradas las micrografías de un área del núcleo del diente y del centro del anillo del engranaje, respectivamente. Se puede observar que el nucleo del diente tiene una microestructura constituida de martensita revenida, austenita retenida (constituyente más claro) y algunas inclusiones dispersas. La existencia de una matriz martensítica en esta área indica que el acero es de alta templabilidad. De acuerdo con Edorgan y Tekeli (2003), en el caso que el acero presente baja templabilidad, seria constituido principalmente de ferrita y una pequeña cantidad de perlita en el núcleo, dependiendo de la velocidad de enfriamiento. Fue verificado que en el centro del anillo la microestructura es más heterogénea con la presencia de bainita, martensita y austenita retenida.
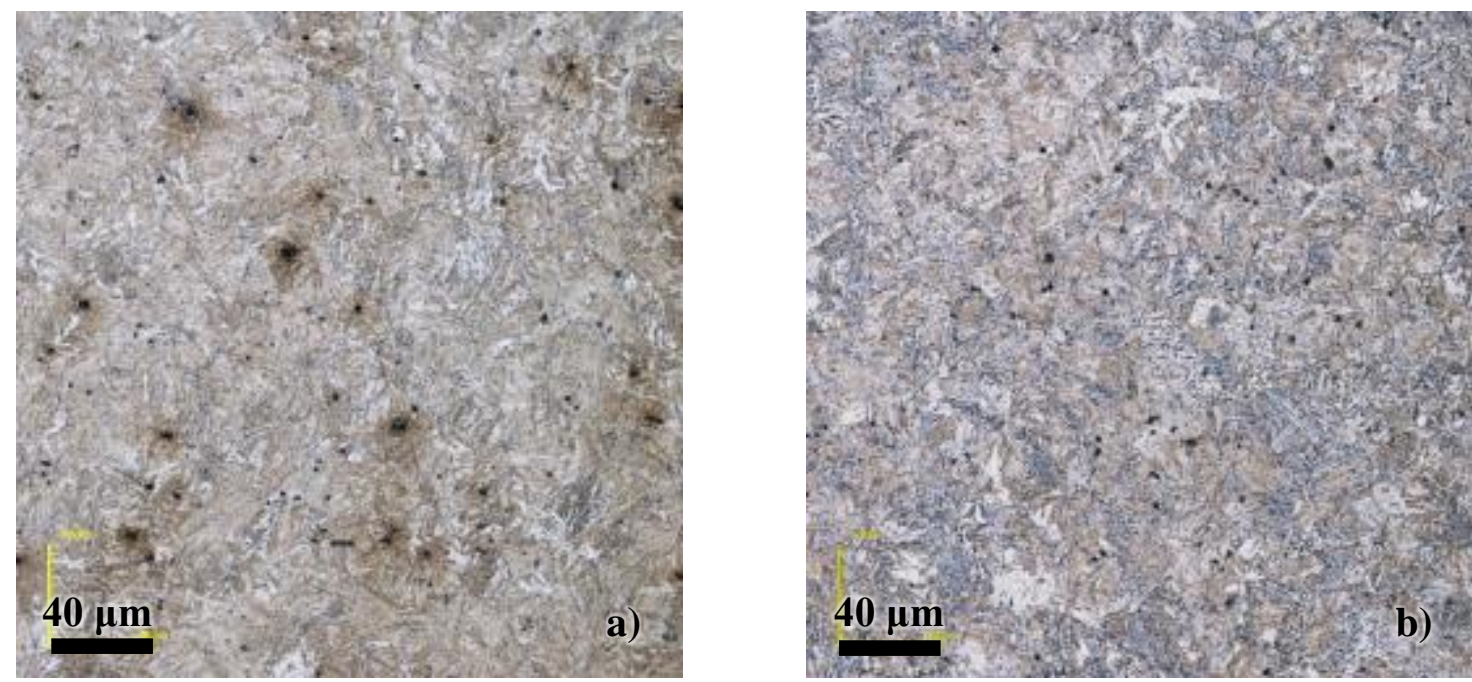

Figura 4: Micrografías obtenidas después del ataque químico con Nital 2\% de las regiones del a) anillo y del b) núcleo.

En la Figura 5 se presenta un montaje de las micrografías de la superficie del engranaje. Fue confirmado que el gradiente de carbono introducido durante la cementación resultó, después del templado, en un gradiente de la microestructura martensítica.

Como puede ser observado en la Figura 5, la superficie presenta una microestructura martensítica que, con el aumento de la distancia de la superficie varia gradualmente para una martensita de bajo porcentaje de carbono. Además, se puede observar la presencia de otros constituyentes microestructurales, como austenita retenida e inclusiones.

En esta misma área fueron observadas tres grietas, las cuales están indicadas como 1, 2 y 3 en la Figura 3. En las Figuras 6 y 7 son presentadas micrografías de las regiones con estas grietas. Como puede ser verificado en la Figura 6, estas grietas son superficiales y que solamente la grieta indicada como 1 del diente 1 presenta ramificaciones. En la micrografía de la Figura 7 se observa esta grieta con mayor aumento, se puede notar que la grieta se propaga a lo largo de los límites de grano de la austenita primaria, siendo clasificado como grieta intergranular. Fue confirmado que las otras grietas también siguieron el mismo camino durante su propagación.

Persp. Online: exatas \& eng., Campos dos Goytacazes, 27 (10) 27 - 41 - 2020

seer.perspectivasonline.com.br 


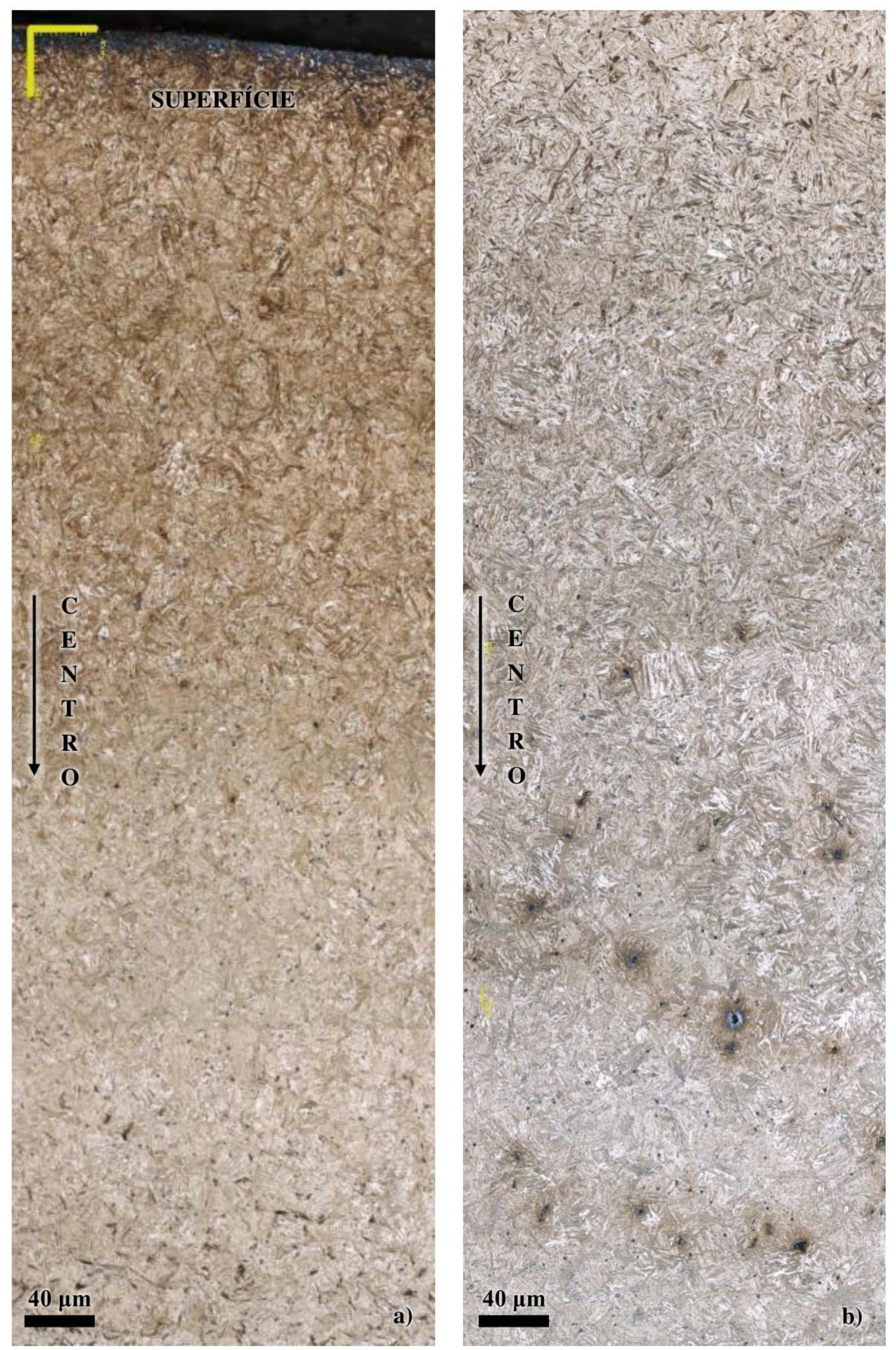

Figura 5: a) Montaje de micrografía desde la superficie del engranaje en dirección al centro; b) continuación

Persp. Online: exatas \& eng., Campos dos Goytacazes, 27 (10) 27 - 41 - 2020 seer.perspectivasonline.com.br 
De acuerdo con Hyde (1992), la grieta que se propaga a lo largo de los límites de grano de la austenita primaria es casi un modo de fractura universal de las capas de superficies tratadas con alto carbono de aceros cementados.

Aunque las grietas intergranulares formadas en los límites de la austenita primaria de las superficies cementadas dominen la iniciación de grietas de fatiga por flexión en el pie del diente y la propagación de grietas inestables de aceros cementados. Este mismo trabajo mostró que tensiones que exceden el límite de resistencia a la fatiga del material son responsables por el surgimiento de grietas intergranulares en la superficie.
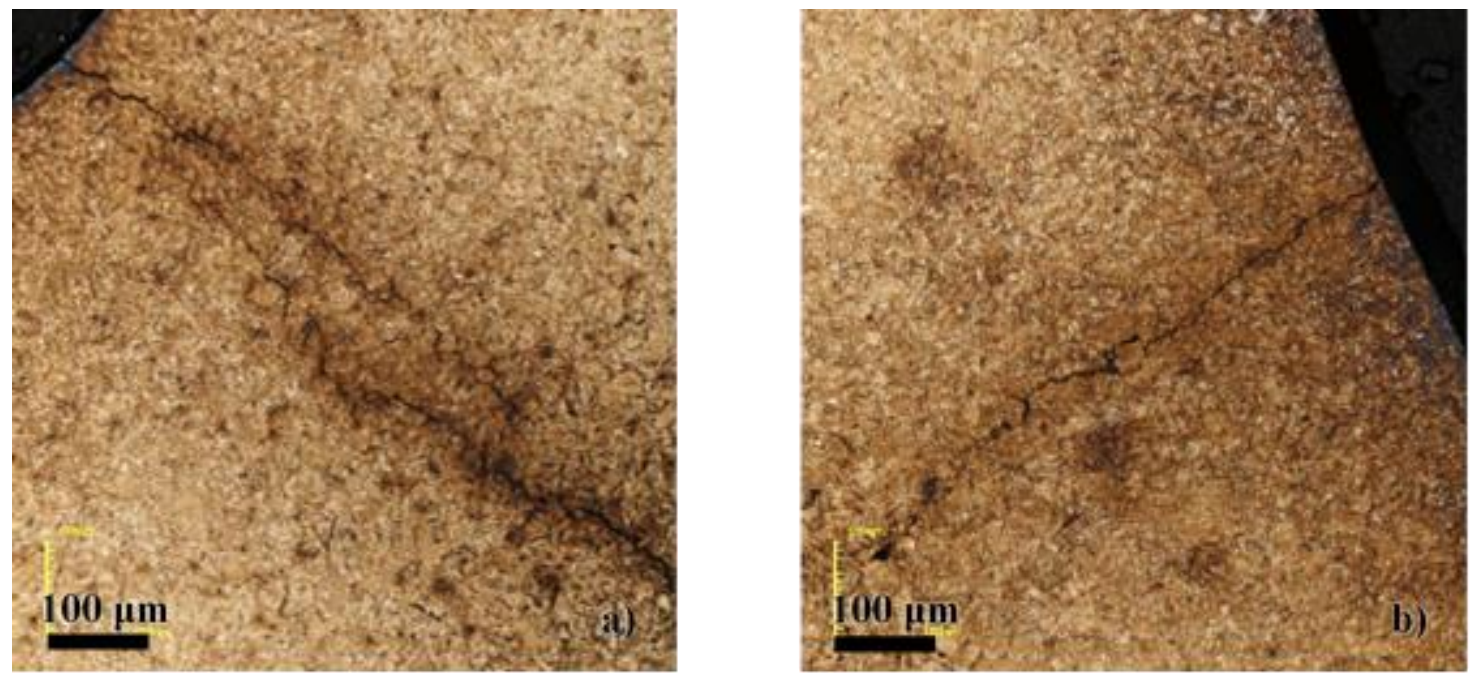

Figura 6: Grietas superficiales encontradas en el diente 1: a) región 1; b) región 2
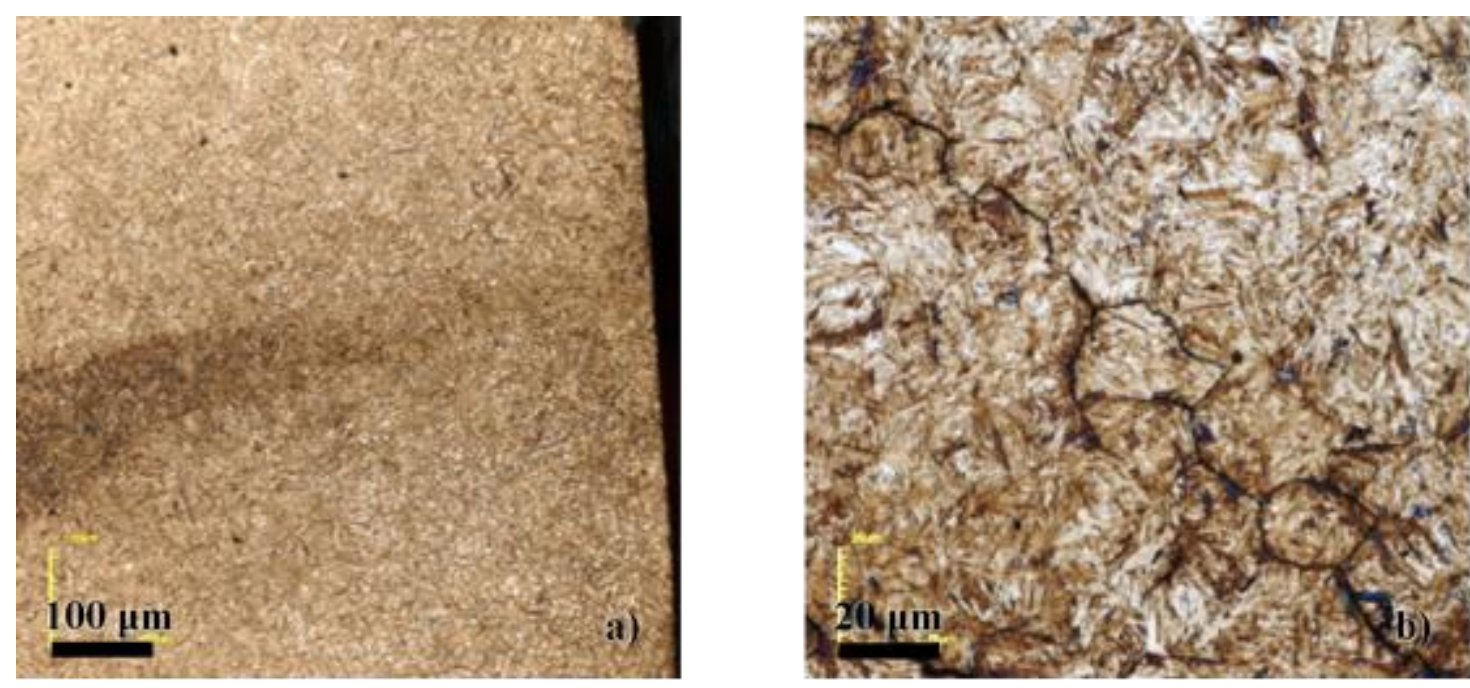

Figura 7: a) Grieta superficial encontradas en el diente 2 en la región 3; b) grieta superficial encontrada en el diente 1 de la región

De acuerdo con Shea (1978) las grietas intergranulares son relativamente pequeñas, estas contornan entre dos y tres granos de la austenita primaria y luego son interrumpidas. Sin embargo, fue observado que el tamaño del camino seguido por la grieta intergranular encontrado en el engranaje estudiado fue mucho mayor. Este autor supuso que los caminos seguidos son pequeños debido a la formación de una zona plástica menor que el tamaño de grano en la punta de la grieta intergranular y por transformación inducida por la deformación de la austenita retenida en la zona plástica. De esta manera, se entiende que, posiblemente, la

Persp. Online: exatas \& eng., Campos dos Goytacazes, 27 (10) 27 - 41 - 2020

seer.perspectivasonline.com.br 
zona plástica formada era mayor que el tamaño del grano y que la microestructura tiene un pequeño porcentaje de austenita retenida o que la deformación no fue suficiente para causar una transformación inducida por deformación.

\subsection{Perfil de la microdureza en los dientes del engranaje}

La Figura 8 presenta el perfil de la microdureza Vickers de los dientes del engranaje.

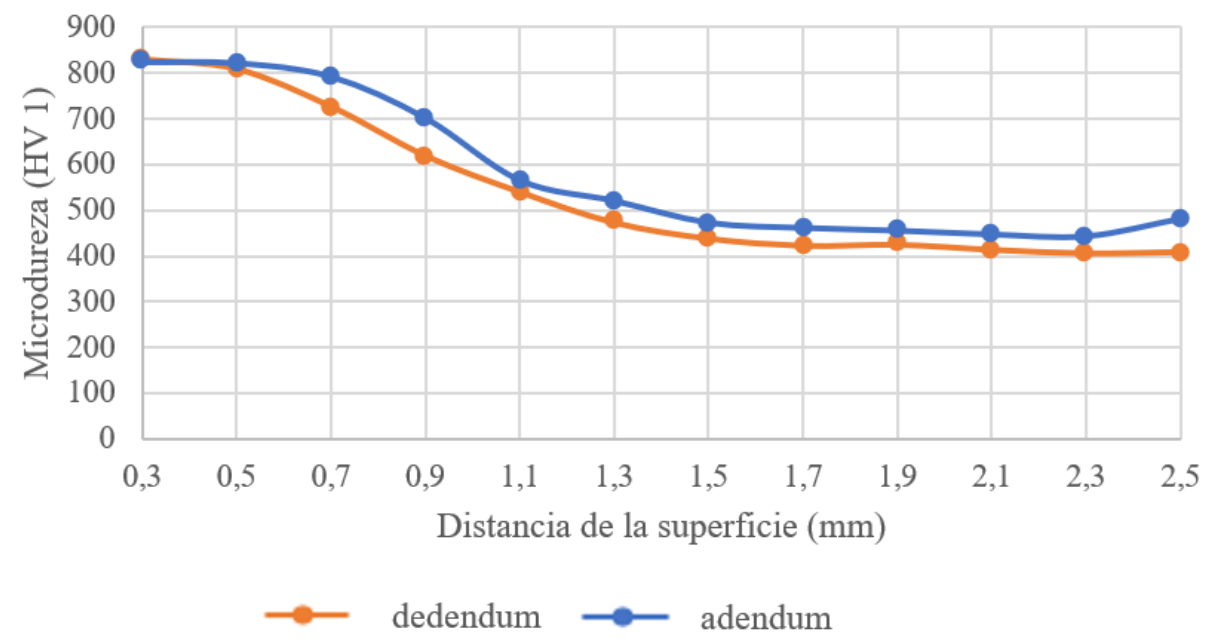

Figura 8: Perfil de la microdureza Vickers al largo de la superficie del diente del engranaje en las regiones del dedendum y del adendum.

Puede ser observado que el valor máximo fue 835 HV1 en la superficie del dedendum, que la microdureza media fue $562 \pm 151 \mathrm{HV} 1$ y que la superficie endurecida presenta una profundidad un poco mayor que 1,0 mm. La norma ANSI/AGMA 2001 D04 estipula que, de acuerdo con el espesor del diente, la profundidad máxima de la superficie endurecida debe ser de 1,23 $\mathrm{mm}$. Con relación al valor mínimo de la profundidad de endurecimiento, de acuerdo con esta norma, establecida por el paso diametral siendo 0,44 $\mathrm{mm}$. De esta manera, se confirma que el valor de la medida de la superficie endurecida se encuentra de acuerdo con los valores mínimo y máximo de la norma.

\subsection{Dureza}

En la Tabla 4 son presentados valores de la dureza medidas en el núcleo del diente y en la superficie endurecida por cementación, así como también los valores especificados por la norma ANSI/AGMA 2001 D04. El núcleo del diente y la superficie endurecida por cementación presentan valores medios de la dureza de $34 \pm 1$ y $57 \pm 1$, respectivamente. La norma mencionada especifica que, para el caso de engranajes de aceros endurecidos por tratamiento termoquímico de cementación debe tenerse una dureza mínima en el núcleo (centro del diente en la altura del diámetro de la raíz) de 21 HRC y exige que estos valores de la dureza en la superficie estén entre 55-64 HRC para aceros endurecidos por tratamiento termoquímico de cementación. Por lo tanto, se puede afirmar que los resultados de dureza encontrados en el engranaje estudiado están en concordancia con los valores establecidos por la norma.

Persp. Online: exatas \& eng., Campos dos Goytacazes, 27 (10) 27 - 41 - 2020 seer.perspectivasonline.com.br 
Tabla 4: Dureza HRC del núcleo y de la superficie endurecida por cementación

\begin{tabular}{|c|c|c|c|c|c|c|c|c|}
\hline \multirow{3}{*}{ Región analizada } & \multicolumn{6}{|c|}{ Dureza Rockwell C (HRC) } & \multirow{3}{*}{ Media } & \multirow{3}{*}{$\begin{array}{l}\text { Desviación } \\
\text { estándar }\end{array}$} \\
\hline & AGMA & & & & & & & \\
\hline & 2001-D04 & 1 & 2 & 3 & 4 & 5 & & \\
\hline Núcleo do dente & 21 (mín.) & 33 & 33 & 33 & 34 & 35 & 34 & 1 \\
\hline $\begin{array}{l}\text { Superficie endurecida } \\
\text { por cementación }\end{array}$ & $55-64$ & 56 & 57 & 57 & 58 & 56 & 57 & 1 \\
\hline
\end{tabular}

\subsection{Tensiones de flexión y de contacto admisibles}

Para un acero de grado 1 endurecido por cementación, la norma ANSI/AGMA 2001/D04 establece una tensión admisible de flexión $\left(s_{a t}\right)$ de aproximadamente 379 MPa y una tensión admisible de contacto $\left(s_{a c}\right)$ de $1241 \mathrm{MPa}$. Las tensiones de flexión $\left(\sigma_{t}\right)$ y de contacto $\left(\sigma_{c}\right)$ calculadas para el engranaje fueron de $481 \mathrm{MPa}$ y $1128 \mathrm{MPa}$, respectivamente.

A partir de estos resultados, se puede verificar que $\sigma_{t}>s_{a t}$, siendo muy probable la falla por sobrecarga de este engranaje. Se observó también que los factores de sobrecarga y el factor de calidad influenciaron considerablemente en el resultado de la tensión de flexión.

En el caso que el factor de sobrecarga fuera igual a 1, que, de acuerdo con Hamrock et al. (2014), es apropiado para motores eléctricos, probablemente esta falla no ocurriría, porque se tendría un factor de seguridad de aproximadamente 1,2. En el caso del engranaje el factor de seguridad encontrado fue menor que 1 , indicando la falla del material.

En relación con la tensión de contacto, fue observado que el engranaje presenta una resistencia apropiada a la formación de cavidades con un coeficiente de seguridad de aproximadamente 1,1 y que, por esta razón, estas características no fueron encontradas en el análisis visual del engranaje.

\section{CONCLUSIONES}

En este trabajo fue estudiado la causa de la falla de un engranaje helicoidal perteneciente a un reversor de un motor de combustión interna de tipo marítimo. Después de realizados las análisis, ensayos y cálculos, se puede concluir que:

- Los dientes del engranaje muestran una fractura característica de sobrecarga, y de acuerdo con el análisis metalográfico presenta una superficie endurecida con espesor uniforme.

- El tratamiento termoquímico de cementación originó un gradiente de concentración de carbono. Como consecuencia del templado se formó una martensita de alto carbono en la superficie y de bajo carbono a medida que se aleja de la superficie provocando un endurecimiento mayor en la superficie.

- En la región endurecida, a la altura del diámetro primitivo y del adendo fue observado la presencia de grietas superficiales que se propagan a lo largo de los contornos de grano de la austenita primaria

Persp. Online: exatas \& eng., Campos dos Goytacazes, 27 (10) 27 - 41 - 2020

seer.perspectivasonline.com.br 
- La tensión de contacto determinada es menor que la tensión admisible de contacto del engranaje, la cual indica que el engranaje presenta una resistencia adecuada a la formación de cavidades. Sin embargo, fue verificado que la tensión de flexión es superior a la tensión de flexión admisible recomendada por la norma, estando por tanto susceptible a una falla por sobrecarga debido a un dimensionamiento inadecuado.

\section{REFERENCIAS}

ALBAN, L. E. Systematic Analysis of Gear Failures. Ohio: American Society for Metals. 1985. 232 p.

AMERICAN GEAR MANUFACTURES ASSOCIATION. ANSI/AGMA 2001-D04: Fundamental Ranting Factors and Calculation Methods for Involute Spur and Helical Gear Teeth. Alexandria, 2004.

AMERICAN GEAR MANUFACTURES ASSOCIATION. ANSI/AGMA 908-B89: Geometry Factors for Determining the Pitting Resistance and Bending Strength of Spur, Helical, Herringbone Gear Teeth. Alexandria, 1989.

AMERICAN SOCIETY FOR METALS. Handbook. Properties and Selection: Irons, Steels, And High-Performance Alloys. Materials Park: ASM International, 1990. 1 v.

AMERICAN SOCIETY FOR METALS. Handbook. Failure Analysis and Prevention. Materials Park: ASM International, 1990. $11 \mathrm{v}$.

AMERICAN SOCIETY FOR TESTING AND MATERIALS. ASTM A29: Standard Specification for General Requirements for Steel Bars, Carbon and Alloys, Hot-wrougth. Philadelphia: ASTM; 2015.

AMERICAN SOCIETY FOR TESTING AND MATERIALS. ASTM E18: Standard Test Methods For Rockwell Hardness Of Metallic Materials. Philadelphia: ASTM; 2016.

ASI, O. Fatigue failure of a helical gear in a gearbox. Enginnering Failure Analysis, v. 13, p. 11161125, 2006. DOI: 10.1016/j.engfailanal.2005.07.020.

ASM HANDBOOK COMMITEE. Properties and Selection: Irons, Steels, and High-Performance Alloys. American Society for Metals, v. 1, 1063 p., 1990.

ASM HANDBOOK COMMITEE. Failure Analysis and Prevention. American Society for Metals, v. 11, 1164 p., 2002.

BROOKS, C. R.; CHOUDRY, A. Metallurgical Failure Analysis. Mc-Graw-Hill: New York, 1993.

DAVIS, J. R. Gear Materials, Properties and Manufacture. ASM International: Materials Park. 2002. $339 \mathrm{p}$.

EDORGAN, M.; TEKELI, S. The effect of martensite volume fraction and particle size on the tensile properties of a surface-carburized AISI 8620 steel with a dual-phase core microstructure. Materials Characterization. v. 49. n. 5, p. 445-454, 2003. DOI: 10.1016/S1044-5803(03)00070-6.

FUJITA, K; YOSHIDA, A; NAGAMORI, K. Effect of Hardness on Tooth Strength and Surface Durability and on Failure Modes of Gears. Bulletin of JSME, v. 25, n. 201, p. 452-458, 1982.

HAMROCK, B.; SCHMID, S.; JACOBSON, B. Fundamentals of Machine Elements. Terceira edição. Boca Raton: CRC Press, 2014.

Persp. Online: exatas \& eng., Campos dos Goytacazes, 27 (10) 27 - 41 - 2020

seer.perspectivasonline.com.br 
HYDE, R. S.; R.E. COHEN; MATLOCK D.K.; KRAUSS, G. Bending Fatigue Crack Characterization and Fracture Toughness of Gas Carburized SAE 4320 Steel. SAE Technical Paper 920534. SAE International, 1992. DOI: 10.4271/920534.

KU, P. M. Gear Failure Modes - Importance of Lubrication and Mechanics. ASLE Transactions, v. 19, n. 3, p. 239-249, 2008. DOI: 10.1080/05698197608982799

LIU, H., LIU, H., BOCHER, P., ZHU, C., SUN, Z. Effects of case hardening properties on the contact fatigue of a wind turbine gear pair. International Journal of Mechanical Sciences, v. 141, p. 520527, 2018. DOI: 10.1016/j.jimecsci.2018.04.010

MELCONIAN, S. Elementos de Máquinas. 9ª Edição. Érica Ltda.: São Paulo. 2009. 376 p.

ROY, S.; OOI, G. T. C.; SUNDARARAJAN, S. Effect of retained austenite on micropitting behavior of carburized AISI 8620 steel under boundary lubrication. Materialia, v. 3, p. 192-201, 2018. DOI: 10.1016/j.mtla.2018.08.029.

ROY, S.; SUNDARARAJAN, S. Effect of retained austenite on spalling behavior of carburized AISI 8620 steel under boundary lubrication. International Journal of Fatigue, v. 119, p. 238-246, 2019. DOI: 10.1016/j.ijfatigue.2018.10.001.

SHEA, M. M. Impact Properties of Selected Gear Steels. Technical Paper 780772. SAE International, 1978. DOI: 10.4271/780772.

SHIGLEY, E.; MISCHKE, R.; BUDYNAS, G. Projeto de Engenharia Mecânica. $7^{\text {a }}$ Edição. Porto Alegre: Bookman, 2008. 957 p.

ZACCONE, M.A.; KELLEY, J.B.; KRAUSS, G. Strain Hardening and Fatigue of Simulated Case Microstructures in Carburized Steel. In: KRAUSS, G. (Org.). Carburizing: Processing and Performance. Materials Park: ASM International. 1989. p 249-265. 